\title{
Insulin resistance and the regulation of vascular tone: is insulin a vasodilator?
}

\author{
U M Vischer \\ Division de Biochimie Clinique, Centre Médical Universitaire, Geneva, Switzerland \\ (Correspondence should be addressed to U M Vischer, Division de Biochimie Clinique, Centre Médical Universitaire, 1 Rue Michel Servet, \\ 1211 Geneva 4, Switzerland)
}

Insulin resistance is a key element in the pathogenesis of non-insulin-dependent diabetes mellitus (NIDDM). Insulin resistance is usually assessed by measurements of insulin-induced glucose uptake, mainly by skeletal muscle, using the euglycemic hyperinsulinemic clamp technique. This involves a constant insulin infusion which stimulates glucose uptake. A variable glucose infusion is regularly adjusted to maintain a constant blood glucose level, i.e. to compensate for tissue glucose uptake. The amount of glucose infused at a given insulin concentration under steady state conditions is used as an index of insulin sensitivity. Insulin increases glucose extraction by skeletal muscle cells, but may also enhance glucose delivery via increased blood flow. This hypothesis was proposed in 1990 by Baron's group (1). Their experimental design involved catheterization of the femoral artery and vein. Measurements of leg blood flow (by thermodilution) and of the arteriovenous glucose difference were performed, and the leg glucose uptake was determined as the product of these two parameters. It came somewhat as a surprise to find that insulin-induced glucose uptake was associated with a striking increase in leg blood flow; the insulin dose-responses of blood flow and glucose extraction were very similar. These findings raised the possibility that insulin is a vasodilator hormone. In addition, the effect of insulin on leg blood flow was blunted in obese insulin-resistant patients, suggesting that vascular (in)sensitivity is a component of insulin resistance.

The mechanism of insulin-induced vasodilation has now become a hotly debated issue. Is there a direct effect of insulin on the vascular wall, or does insulin act indirectly via a metabolic intermediate? Is blood flow (dys)regulation really involved in the pathogenesis of insulin resistance? Attention has focused on the regulation of endothelium-dependent vasodilation. In response to neurotransmitters such as acetylcholine, endothelial cells produce the vasodilator nitric oxide (NO), which acts in a paracrine manner on underlying smooth muscle cells to regulate contraction. NO activates a membrane guanylate cyclase, resulting in increased cGMP levels and activation of cGMP-dependent protein kinases (2). Using the leg perfusion system, the Baron group found that administration of metacholine (an acetylcholine analog) caused an expected dosedependent increase in leg blood flow. Simultaneous administration of insulin had a strong potentiating effect on the response to metacholine. Furthermore, the administration of L-NMMA, a specific inhibitor of NO synthase, abolished insulin-induced vasodilation (3). This finding was confirmed by others using a forearm perfusion technique (4). The response to metacholine was severely blunted in patients with insulin resistance or NIDDM, whereas the response to sodium nitroprusside, a direct activator of guanylate cyclase, was unaffected (5). These findings strongly suggest that insulin resistance is associated with impaired endothelium-dependent vasodilation. Similarly, the insulin resistance associated with insulin-dependent diabetes mellitus (IDDM) has also been attributed to impaired insulin-dependent NO-mediated vasodilation (6).

Although theoretically attractive, these observations have not met with unanimous acceptance. Yki-Järvinen and coworkers studied the response to insulin infusion in the forearm. They found increased blood flow only at high, supraphysiological insulin concentrations, whereas the increase in arterio-venous glucose difference was observed with lower doses (7). In agreement with the Baron group, they also reported impaired insulin sensitivity in IDDM patients. However, the impairment was attributed to a blunted increase in glucose extraction (i.e. arterio-venous glucose difference), while even at high insulin doses forearm blood flow was unaffected in diabetic patients relative to controls (8). The discrepant results from these two research teams, particularly those on the effect of insulin in normal individuals, have not been elucidated. Differences in blood flow measurement techniques and sites of measurement (leg versus forearm) may play a role. The timing of blood flow measurements relative to the start of the insulin infusion was also different (longer in the Baron studies), suggesting that the insulin effect at low doses may be a delayed response. The mechanism of insulin-induced NO synthesis may involve direct activation of an endothelial insulin receptor. This hypothesis attributes a causal effect to hyperinsulinemia per se, and further implies that insulin resistance affects the endothelium as well as other insulinsensitive tissues. Insulin receptors have been described in some endothelial cell culture systems, but the issue is confused by the heterogeneity of endothelial cells in different sites in vivo. Further, these insulin receptors may relate to transendothelial insulin transport (i.e. transcytosis) 
rather than metabolic activation. Alternatively, insulininduced NO synthesis could be mediated by an indirect metabolic effect. Insulin inhibits lipolysis in adipose tissue, resulting in decreased free fatty acid (FFA) levels. Could FFAs inhibit acetylcholine-mediated vasodilation? Could insulin act by decreasing FFAs? In a recent paper (9), the effect of FFAs was tested by administration of Intralipid, a triglyceride preparation used in parenteral nutrition, and heparin which activates lipoprotein lipase, thus converting the infused triglycerides into FFAs and glycerol. Tested at two different dosages, the Intralipid/heparin combination caused a striking inhibition of metacholine-induced increase in leg blood flow. In contrast, the response to sodium nitroprusside was unaffected, again suggesting that FFAs specifically inhibit endothelium-dependent vasodilation. Control experiments with Intralipid alone (without heparin) ruled out the possibility that triglycerides, rather than FFAs, are involved.

To test whether endogenous FFAs could achieve the same inhibitory effect, the authors attempted to increase endogenous FFA levels by somatostatin infusions. Somatostatin reduces insulin levels, which causes increased lipolysis (and therefore raises FFA levels), but also inhibits secretion of several other peptide hormones such as glucagon. Similar to Intralipid/heparin, somatostatin infusion inhibited the vasodilatory response to metacholine, but not to sodium nitroprusside. In a control experiment, somatostatin was infused together with replacement doses of insulin. The metacholine-induced vasodilation was not different from saline-infused controls, arguing against an effect of somatostatin on glucagon or other peptide hormones. However, this experiment fails to differentiate between insulinopenia (i.e. a direct effect of insulin) and an indirect effect mediated by FFAs in somatostatin inhibition of NOdependent vasodilation. Note that whether the effect of insulin is direct or not, the somatostatin experiments suggest that insulin regulates NO-mediated vasodilation even at normal, 'physiological' concentrations, since it is impaired by decreasing insulin below those levels. If insulin-mediated vasodilation is indeed mediated by inhibition of lipolysis, it should be prevented by coinfusion of Intralipid during a euglycemic clamp. In fact such an experiment was performed by Vollenweider et al. (10) who failed to observe an effect of Intralipid on insulinmediated vasodilation. However, this negative result may be due to the use of plethysmography for blood flow measurements, which is not a very sensitive method.

Taken together the available data strongly suggest that insulin regulates NO-mediated vasodilation. One possible mechanism is indirect via the inhibition of lipolysis and the consequent decrease in FFA circulating levels. However, these experiments do not rule out an additional direct effect of insulin on endothelial cells. Endothelium-independent mechanisms could also be involved. Although this contention is still controversial, impaired NO-mediated vasodilation may participate in the pathogenesis of insulin resistance. The concept that regulation of blood flow determines glucose utilization could provide at least a partial explanation for the effect of anti-hypertensive drugs on glucose metabolism. Glucose tolerance is impaired by beta blockers and improved by angiotensin converting enzyme inhibitors: these drugs are peripheral vasoconstrictors and vasodilators respectively. In addition to its role in the regulation of vascular tone, NO also inhibits platelet aggregation and surface expression of endothelial adhesion molecules, and may thus exert a protective role against atherosclerosis. An impairment in insulin-mediated NO production may provide one explanation for the well known association between insulin resistance and an increased risk of macrovascular disease.

\section{References}

1 Laakso M, Edelman SV, Brechtel G \& Baron AD. Decreased effect of insulin to stimulate skeletal muscle blood flow in obese man. A novel mechanism for insulin resistance. Journal of Clinical Investigation 199085 1844-1852.

2 Vane JR, Anggard EE \& Botting RM. Regulatory functions of the vascular endothelium. New England Journal of Medicine 1990323 27-36.

3 Steinberg HO, Chaker H, Leaming R, Johnson A, Brechtel G \& Baron AD. Obesity/insulin resistance is associated with endothelial dysfunction. Implications for the syndrome of insulin resistance. Journal of Clinical Investigation 199697 2601-2610.

4 Scherrer U, Randin D, Vollenweider P, Vollenweider L \& Nicod P. Nitric oxide accounts for insulin's vascular effects in humans. Journal of Clinical Investigation 199494 2511-2515.

5 Steinberg HO, Brechtel G, Johnson A, Fineberg N \& Baron AD. Insulin-mediated skeletal muscle vasodilation is nitric oxide dependent. A novel action of insulin to increase nitric oxide release. Journal of Clinical Investigation 199494 1172-1179.

6 Baron AD, Laakso M, Brechtel G \& Edelman SV. Mechanism of insulin resistance in insulin-dependent diabetes mellitus: a major role for reduced skeletal muscle blood flow. Journal of Clinical Endocrinology and Metabolism 199173 637-643.

7 Utriainen T, Malmström R, Mäkimattila S \& Yki-Järvinen H. Methodological aspects, dose-response characteristics and causes of interindividual variation in insulin stimulation of limb blood flow in normal subjects. Diabetologia 199538 555-564.

8 Mäkimattila S, Virkamäki A, Groop PH, Cockcroft J, Utriainen T, Fagerudd J \& Yki-Järvinen H. Chronic hyperglycemia impairs endothelial function and insulin sensitivity via different mechanisms in insulin-dependent diabetes mellitus. Circulation 199694 1276-1282.

9 Steinberg HO, Tarshoby M, Monestel R, Hook G, Cronin J, Johnson A, Bayazeed B \& Baron AD. Elevated circulating free fatty acid levels impair endothelium-dependent vasodilation. Journal of Clinical Investigation 1997100 1230-1239.

10 Vollenweider L, Tappy L, Owlya R, Jéquier E, Nicod P \& Scherrer U. Insulin-induced sympathetic activation and vasodilation in skeletal muscle. Diabetes $199544641-645$. 